$\underline{\text { Review }}$

\title{
Candida auris: A brief review
}

\author{
LSM Sigera $^{1}$, MN Jayawardena ${ }^{2}$, H Thabrew $^{1}$, PI Jayasekera ${ }^{1}$ \\ Sri Lankan Journal of Infectious Diseases 2020 Vol.10 (1):2-8
}

DOI: http://dx.doi.org/10.4038/sljid.v10i1.8270

\begin{abstract}
Candida auris is an emerging fungal pathogen that has caused global concern because of its multidrug-resistant nature and ability to cause uncontrollable nosocomial outbreaks. It is frequently misidentified as other Candida species by routine laboratory procedures, necessitating modern molecular diagnostic techniques for diagnosis. A relatively high mortality rate associated with this organism highlights the validity of implementation of strict infection control and preventive measures in the presence of infected or colonized patients.
\end{abstract}

Keywords: Candida auris, Multidrug resistance, Nosocomial outbreaks, Laboratory misidentification, Infection control and prevention.

\section{Introduction}

Candida auris is an emerging multidrug resistant pathogen which was first isolated in 2009 from a Japanese patient. ${ }^{1}$ It has thereafter been reported in several other countries across the globe including India, Pakistan, Kuwait, Canada, Israel, Oman, South Africa, Colombia, the United States, South Korea, Norway, the United Kingdom, Spain, and Germany. ${ }^{2}$ In addition to wellknown four different geographical clades, South Asian (I), East Asian (II), African (III) and South American (IV), a study in Iran has recently described a potential fifth clade. ${ }^{3}$ The reported mortality of Candida auris shows great variation in different geographical locations and some reports show it could extend up to $72 \% .^{1}$

Candida auris has become a major concern across the globe due to its multidrug resistant nature, its potential to cause nosocomial outbreaks and the possibility of misidentification by routine laboratory procedures. ${ }^{1}$

\footnotetext{
${ }^{1}$ Department of Mycology, Medical Research Institute, Colombo, Sri Lanka

${ }^{2}$ National Cancer Institute, Maharagama, Sri Lanka

Address for correspondence: Dr LSM Sigera, Department of Mycology, Medical Research Institute, Colombo, Sri

Lanka. Telephone: +0094777008739 Email: sshamithra@yahoo.com

(iD) https://orcid.org/0000-0002-5519-7619

Received 25 October 2019 and revised version accepted 3 March 2020

(c) (†)

This an open-access article distributed under the terms of the Creative Commons Attribution License, which permits unrestricted use, distribution, and reproduction in any medium, provided the original author and source are credited.
} 


\section{Risk factors and infection}

Neutropenia, diabetes mellitus, exposure to broad-spectrum antibiotics, presence of a central venous catheter, hemodialysis, parenteral nutrition, blood transfusion, admission to intensive care, previous antifungal agents within 30 days, surgery within 30 days, concomitant bacteraemia, concomitant candidemia, indwelling urinary catheter, candiduria and chronic kidney disease have been identified as risk factors for the infection which are also common to other Candida species causing infections. ${ }^{4}$

This pathogen has been identified as responsible for fungemia, surgical wound infection, urinary tract infection, respiratory tract infection, ear infection, central nervous system infection, skin and soft tissue infection, bone infection and myocarditis. ${ }^{5}$

\section{Diagnosis of infection}

Accurate identification of $C$. auris requires sophisticated laboratory methods such as sequencing, improved mass spectrometry, or a VITEK 2 version $8 .^{6}$ Genetic sequencing of different loci specific to $C$. auris such as D1/D2 region of the $28 \mathrm{~S}$ rDNA or ITS of rDNA, is also an efficient method of identification and differentiation of geographic clades. ${ }^{6}$ Although older versions of VITEK 2 (bioMérieux, Marcy-l'Étoile, France) have misidentified $C$. auris as $C$. haemulonii or $C$. famata, the VITEK 2 version 8.01 is capable of differentiating $C$. auris from other Candida species. ${ }^{4,6}$ In the presence of specific spectra of $C$. auris in the database of Matrix-assisted laser desorption ionization-time of flight mass spectrometry (MALDI-TOF MS), C. auris has been distinguished from closely related candida species. ${ }^{1}$

Most routine laboratories in resource poor settings do not identify Candida isolates up to species level which results in non-detection of patients with $C$. auris infections. ${ }^{6}$ In addition, identification remains difficult and problematic in settings where the diagnostic methods mentioned above are not available. ${ }^{4,7}$ Phenotypic and biochemical techniques used in conventional laboratories in the absence of molecular diagnostics provide inconclusive results leading to misidentification $C$. auris. ${ }^{4}$ For example, microscopically, $C$. auris forms oval or elongated yeast cells without hyphal or pseudo hyphal forms indistinguishable from other yeast species. ${ }^{1}$ In addition, they form pink to beige colored colonies on chromogenic agar mimicking other candida species such as $C$. parapsilosis. ${ }^{1,4}$ Consequently, $C$. auris is frequently misidentified by routine conventional phenotypic and biochemical methods as $C$. haemulonii, $C$. sake, C. famata, C.parapsilosis, C.lusitaniae, Saccharomyces species, Rhodotorula glutinis, and Rhodotorula mucilaginosa.,8 Most commercial systems frequently used in laboratories also misidentify $C$. auris. ${ }^{4}$ For example, it is reported that it has been misidentified as $R$. glutinis or $C$. sake by API20C (bioMérieux) while Auxacolor (Bio-Rad, Hercules, CA, USA) misidentifies it as $S$. cerevisiae. ${ }^{4,6}$

C. auris produces special features in certain biochemical testing methods which would assist accurate identification in laboratories using phenotypic/biochemical identification methods. $C$. auris tolerates both $42{ }^{\circ} \mathrm{C}$ temperature and $10 \% \mathrm{NaCl}$ and is intolerant to $0.01 \%$ and $0.1 \%$ cycloheximide. ${ }^{4,6}$ In such settings, drug resistant Candida isolates producing beige to pink colonies on chrome agar which tolerate $42{ }^{\circ} \mathrm{C}$ and $10 \% \mathrm{NaCl}$ and do not tolerate cycloheximide 
$(0.01 \%, 0.1 \%)$ should be suspected as Candida auris and forwarded to reference laboratories for molecular identification.

\section{Resistance profile}

Multidrug resistance has been well recognized in $C$. auris and according to data from three continents, almost forty percent of $C$. auris are multidrug resistant. ${ }^{9}$ Although not fully understood, multiple resistance mechanisms such as Erg 11 mutation, $F K S$ mutation are likely to be involved in this phenomena. ${ }^{6}$ In addition, resistance development under the influence of selective pressure is also observed in $C$. auris. ${ }^{9}$ Although MIC break points for $C$. auris have not yet been established, ${ }^{9}$ tentative breakpoints have been proposed by CDC based on data of other Candida species: $\geq 32$ for fluconazole, $\geq 2$ for amphotericin $\mathrm{B}$ (or $\geq 1.5$ if using Etest), $\geq 4$ for anidulafungin and micafungin, and $\geq 2$ for caspofungin. ${ }^{6}$

Currently, up to $90 \%$ of strains have been reported as resistant to fluconazole while $30-40 \%$ and just $5-10 \%$ have been reported as resistant to amphotericin B and the echinocandins respectively. 9 A low level of susceptibility to itraconazole, voriconazole, and isavuconazole has also been described. ${ }^{1}$

\section{Treatment}

With the available resistance data profile, echinocandins are recommended as first line empirical treatment. ${ }^{6}$ Amphotericin B is regarded as an alternative to the echinocandins. ${ }^{9}$ Unfortunately, these antifungals are not widely available and are expensive in resource poor countries. ${ }^{6,8}$

It should be noted that the penetration of echinocandins to the central nervous system is not adequate and its use is questionable in central nervous system infection by $C$. auris. ${ }^{8}$ Inadequate amount of active drug can be found in the urine, thereby reducing its effectiveness in urinary tract infections. ${ }^{1}$ Although there is limited information regarding susceptibility profile of 5flucytosine, in these circumstances combination of amphotericin B with 5-flucytosine has been suggested. ${ }^{1}$ A case report of $C$. auris shunt infection successfully treated using intra-ventricular caspofungin is also noteworthy. ${ }^{8}$

The CDC recommends amphotericin B deoxycholate as first line therapy for neonates and infants under 2 months infected with Candida auris. An echinocandin is infrequently recommended in this group and given only after exclusion of central nervous system infection. ${ }^{6}$ Synergistic antifungal sensitivity testing has suggested that the combination of voriconazole and micafungin is effective in infections caused by multi-resistant isolates. ${ }^{5}$

Empirical treatment should be tailored as soon as possible according to antifungal sensitivity testing results. ${ }^{4}$ Patients should be closely followed up and monitored because treatment failure has been documented. ${ }^{6}$ Depending on clinical presentation, removal of lines and catheters and surgical debridement are advised in combination with antifungal therapy. ${ }^{6}$ The exact duration of treatment is similar to that for other Candida species. ${ }^{4}$ 


\section{Infection prevention and control}

The association of $C$. auris with a high degree of antifungal resistance and high mortality rates necessitates the swift implementation of infection control and prevention methods to curtail its transmission. ${ }^{1}$ C. auris has strong potential for transmission in healthcare settings, resulting in large healthcare associated out breaks. ${ }^{6}$ This is further facilitated by its ability to persist in the environment, irrespective of implementation of all preventive measures. ${ }^{1}$

Although the exact mode of transmission is unknown, it is believed that transmission could be directly through fomites such as blood pressure cuffs, stethoscopes or indirectly through hands of health care workers. ${ }^{4,9}$ According to route cause analysis of an outbreak in London a minimum of more than a four hour contact period with an infected patient or contaminated environment is needed for transmission. ${ }^{10}$

Guidelines have been released by different countries with recommendations related to contact precautions, isolation of patients and cleaning of equipment and the environment in the presence of an infected patient. ${ }^{1,6,9,11}$ Most recommendations in these guidelines have been extrapolated from information on guidelines for resistant bacteria such as carbapenem resistant Enterobacteriaceae (CRE) and methicillin resistant Staphylococcus aureus (MRSA). ${ }^{1,6}$

\section{Patient care}

The detection of $C$. auris should be reported immediately to the infection control team. ${ }^{12}$ The patient or colonized individual should be placed under "contact precautions". ${ }^{1,9}$ Infected or colonized patients should be isolated in a single room with en-suite facilities to prevent horizontal transmission to other patients.,12 Under ideal conditions this room should have negative pressure, an ante room and en-suite bathroom and toilet facilities. ${ }^{12}$ In the absence of en-suite washing facilities, they should be provided with separate dedicated washing and toilet facilities. ${ }^{12}$ Visitors should be informed about $C$. auris through the visible flag system at the entry. ${ }^{12}$ It is recommended to cohort patients with $C$. auris in the same room if single rooms are unavailable. ${ }^{6}$ Sharing of equipment used for infected or colonized patients should be avoided and single-patient use devices are recommended (blood pressure cuffs and pillows), especially in outbreak situations. ${ }^{11}$ Disposable biomedical products and equipment are preferred to reusable products and equipment during patient care. ${ }^{12}$ Visitors of affected individuals should be informed about $C$. auris infection and preventive measures used for control of transmission, ${ }^{4}$

\section{Standard precautions and personal protective equipment}

Standard precautions should be strictly followed by health care workers (HCW) and hands should be cleaned with soap and water followed by alcohol rubs on dry hands. ${ }^{4}$ Personal protective equipment (gloves, apron, gowns) should be worn after hand washing and before entering the patient area. ${ }^{11}$ They are removed and discarded in the room followed by thorough hand cleaning when the HCW leaves the room. ${ }^{4}$ Although it is not required routinely, if there is a risk of spillage or splashes, visors and masks should be used. ${ }^{4,11}$ 


\section{Movement and procedures in the health care setting}

If there is an investigating procedure, infected or colonized patients should be included as the last on the list. ${ }^{11}$ When an infected or colonized patient is transferred to another unit, the receiving unit should be informed of the $C$. auris status of the patient to ensure obtaining appropriate precautions. $^{4}$

\section{Environmental and terminal cleaning}

C. auris has been isolated from different contact areas, such as the patient's equipment, furniture, and on air-settle plate sampling, so environmental cleaning is important. ${ }^{1}$ It is recommended to clean the patient's environment daily and conduct terminal cleaning when the patient leaves the room. ${ }^{4}$

$1000 \mathrm{ppm}$ hypochlorite is recommended for environmental cleaning ensuring thorough contact of all surfaces with the disinfectant. ${ }^{1,4}$ Ultraviolet light and vaporized hydrogen peroxide are also effective in environmental cleaning. ${ }^{4}$ In contrast, quaternary ammonium compounds, acetic acid, and ethyl alcohol seem to be less effective. ${ }^{1,4,6}$

Decontamination of reusable equipment, such as pulse oximeter probes and axillary thermometer and mobility aids should be done according to manufacturer's instructions. ${ }^{1,11}$

\section{Waste disposal}

Waste and linen disposal should be conducted as for any other multi-resistant healthcareassociated organism. ${ }^{12}$ Waste and used linen should be disposed into appropriate bags for isolation to avoid environmental contamination. ${ }^{4}$

\section{De-isolation}

A decision on de-isolation of the patient still remains in a dilemma with the evidence of recurrent colonization after antifungal treatment and negative screening. ${ }^{1}$ According to CDC recommendations, a patient who is not on antifungals can be de-isolated if he/she had at least two negative screens taken a week apart. ${ }^{1}$ A series of three negative tests 24 hours apart have also been suggested before de-isolating the patient. ${ }^{4}$ Three consecutive negative screening results obtained during day 3,5, 7 would be sensible to confirm as negative for C. auris. ${ }^{12}$ Thereafter, weekly screening should be performed until discharge. ${ }^{12}$ However, they should be flagged for at least one year after the first negative screening. ${ }^{12}$

\section{Screening}

Development of screening policies should be based on the risk assessment within local units and infection prevalence. ${ }^{1}$ Infected patients with Candida auris and patients transferring from $C$. auris positive hospitals /units or from countries without diagnosed cases (C. auris undetected) should be screened for $C$. auris. ${ }^{1,4}$ In certain units, it is also considered in circumstances when there is a high risk of candidiasis such as HIV/AIDS patients, patients in Intensive Care Units, 
pediatric patients, patients with malignancies and those undergoing hematopoietic stem cell transplants ${ }^{4}$ Although, the axilla and groin have become the most preferred sites for screening, nose, throat, urine/urethral swab, perineum or lower vagina, sputum, endotracheal secretions, drain fluid (abdominal/pelvic/mediastinal), cannula entry sites (if clinically indicated) and wounds are also possible sites ${ }^{4,12}$.

\section{Decolonization}

Although it is difficult, twice daily decolonization of the skin with $2 \%$ chlorhexidine gluconate or aqueous $4 \%$ chlorhexidine and oral decolonization with $0.2 \%$ chlorhexidine mouthwash or $1 \%$ chlorhexidine dental gel is practiced. ${ }^{4}$

\section{Conclusion}

Candida auris is an emerging multidrug resistant yeast which has been reported across the globe. The relatively high mortality rate and high degree of transmissibility associated with this pathogen highlights the requirement of rapid and accurate identification of the pathogen. However, misidentification in the absence of sophisticated laboratory methods, especially in resource poor settings complicates the picture. In order to treat infected patients and implement infection control measures, establishment of diagnostic facilities, at least for high risk groups, and conducting surveillance programmes to determine the disease epidemiology would be crucial.

\section{References}

1. Jeffery-Smith A, Taori SK, Schelenz S, et al. Candida auris: a review of the literature. Clin Microbiol Rev 2018; 31: e0029-17 doi: https://doi.org/10.1128/cmr.00029-17

2. Kordalewska M, Zhao Y, Lockhart SR, et al. Rapid and accurate molecular identification of the emerging multidrug-resistant pathogen Candida auris. Journal of Clinical Microbiology 2017; 55: 2445-2452. doi: https://doi.org/10.1128/JCM.00630-17.

3. Chow NA, de Groot T, Badali H, et al. Potential fifth clade of Candida auris, Iran. Emerging Infectious Diseases 2018; 25:1780-1781. doi: https://doi.org/10.3201/eid2509.190686

4. Sarma S and Upadhyay S. Current perspective on emergence, diagnosis and drug resistance in Candida auris; Infection and Drug Resistance 2017;10:155-165.

doi: https://doi.org/10.2147/idr.s116229

5. Cortegiani A, Misseri G, Fasciana T, et al. Epidemiology, clinical characteristics, resistance, and treatment of infections by Candida auris. Journal of Intensive Care 2018; 6: Article number 69 doi: https://doi.org/10.1186/s40560-018-0342-4.

6. Forsberg K, Woodworth Walters M, et al. Candida auris: The recent emergence of a multidrugresistant fungal pathogen. Medical Mycology 2019; 57:1-12, doi: https://doi.org/10.1093/mmy/myy054

7. Sexton DJ, Kordalewska M, Bentz ML, et al. Direct detection of emergent fungal pathogen Candida auris in clinical skin swabs by SYBR green-based quantitative PCR assay. Journal of Clinical Microbiology, 2018; 56: e01337-18 doi: https://doi.org/10.1128/JCM.01337-18.

8. Singhal T, Kumar A, Borade P, et al. Successful treatment of C. auris shunt infection with intraventricular caspofungin. Medical Mycology Case Reports 2018; 22:35-37. doi: https://doi.org/10.1016/j.mmcr.2018.08.005.

9. Cortegiani A, Misseri G, Giarratano A, et al. The global challenge of Candida auris in the intensive 
care unit. Critical Care 2019; 23:150 doi: https://doi.org/10.1186/s13054-019-2449-y

10. Schelenz S, Hagen F, Rhodes JL, et al. First hospital outbreak of the globally emerging auris in a European hospital. Antimicrobial Resistance \& Infection Control 2016; 19; 5, doi: http://dx.doi.org/10.1186/s13756-016-0132-5

11. Bishop L, Cummins M, Guy R, et al. Guidance for the laboratory investigation, management and infection prevention and control for cases of Candida auris. Public Health England 2017; Public Health England, United Kingdom. PHE publications gateway number: 2016122

12. Kenters N, Kiernan M, Chowdhary A, et al. Control of Candida auris in healthcare institutions. Outcome of an ISAC expert meeting. International Journal of Antimicrobial Agents 2019; 54:400406 doi: https://doi.org/10.1016/j.ijantimicag.2019.08.013 